Journal of Computer Science 8 (10): 1739-1748, 2012

ISSN 1549-3636

(C) 2012 Science Publications

\title{
Multicost Routing Approach in Wireless Ad hoc Networks
}

\author{
${ }^{1}$ Loganathan, D. and ${ }^{2} \mathrm{P}$. Ramamoorthy \\ ${ }^{1}$ Department of CSE, \\ ${ }^{1}$ Hindusthan Institute of Technology, Coimbatore-32, Tamilnadu, India \\ ${ }^{2}$ Dean (Academics), SSIET, Coimbatore-62, Tamilnadu, India
}

\begin{abstract}
Problem statement: A Wireless ad hoc network is a collection of autonomous movable nodes that communicate with each other over wireless links without any static infrastructure. In these networks there is no fixed topology because of the mobility of nodes, interference, multipath propagation and path loss. A variety of routing protocols and algorithm with varying network settings are analyzed to link between the nodes and communicate packets to their destinations. Most of these algorithms are single cost, in the logic that they consign a scalar cost parameter to every link and compute the path that has minimum cost. Although multicost routing, a vector of cost parameters is consigned to each link and the cost vector of a path is well-defined based on the cost vectors of the links that embrace it. Adjustable transmission power control of the nodes with multi cost routing algorithm can support optimizeto acquire the reduced interference and improve the ad hoc network performance. Approach: The link and path of the wireless network is consigned with several cost parameters. Hop count, total interference, node link delay, residual energy of a node and the node transmission power are the cost parameters assigned for link and path of the ad hoc networks. Multicost parameters are combined in different optimization function with respect to various routing algorithm. Results: Simulation and optimization shows multicost SUM/MIN Energy-Interference algorithm with variable transmission power can lead to decrease the interference and improves the overall network performance. Conclusion: The function optimized for wireless ad hoc networks that the Multicost SUM/MIN Energy-Interference algorithm achieves improved performance over than the single cost algorithm.
\end{abstract}

Key words: Wireless ad hoc networks, multicost, transmission power, network performance

\section{INTRODUCTION}

In wireless ad hoc networks, mobile nodes connect with each other using multi hop wireless links. There is no fixed infrastructure such as base stations. Wireless ad hoc networking has been the emphasis of many current research and development determinations for its applications in military, commercial and educational environments such as Wi-Fi connection networks implemented wherever it is required. Transmit the packets between the nodes as relay transmission with intermediate nodes among three nodes shown in the Fig. 1.

Wireless ad hoc networks typically comprise of battery based operating devices that communicate the data over the wireless communication media. These devices need to be energy preserving and to be life of the battery is maximized. The energy for broadcast of a packet in the wireless channel remains somewhat substantial and may produce to be the highest energy consuming component of the networks. Wireless communication concept concentrates and research continues to decrease the energy consumption for usage of node capacity and storage for the mobile nodes and the performance of wireless ad hoc networks moreover restricted with interference and packet delivery ratio considerations.

Most of the existing protocols and algorithms are single cost concept where all the path of network is categorized by a scalar, which is the sum of the cost that described every one of its essential links. The link cost enables the task of numerous network parameters even still which are scalar. The net results of the single cost routing algorithm estimate the path that has minimum cost for each pair of source-destination nodes. Also single cost routing algorithms is not possible to optimize the network performance relating to common cost functions and do not merely support with QoS variation.

In multicost routing, each link is consigned with parameters of cost vector and path cost vector is constructed on cost vectors of links on the network. From each source to destination pair have to pick the optimal path using optimization function from set of candidate non dominated paths. 


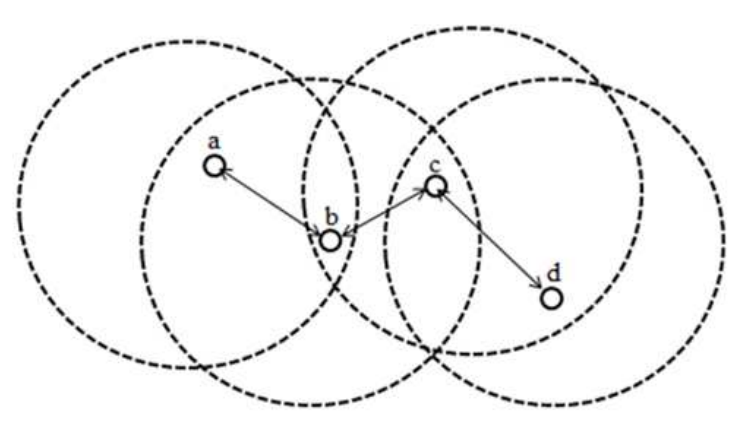

Fig. 1: Wireless Ad hoc networks with intermediate nodes

The hop count, total interference instigated by a packet communication, node link delay, residual energies and the transmission power (Karagiorgas et al., 2007) are cost parameters and used in joint power control $(\mathrm{Li}$ and Ephremides, 2007) of the wireless ad hoc networks.

The new descriptions of link and path cost parameters that are suitable for implementing better routing algorithms. The cost parameters are carefully examined and construct the multicost routing algorithm. Transmission power of the nodes can regulate their minimum necessary transmission power for coherent reception at the receiver node. Whereas the static transmission power of the network consumes extra power than actual power required. The higher energy consumption is lead to increased network interference. Though, reduce the energy consumption and therefore to reduce the interference of the network.

The energy and interference aware routing protocols are assessed that of the migration problem with boundary constraints. The transmission of the network starts with certain number of packets to be routed with optimal path consumed certain amount of energy in each node. And with less amount of delay to serve the packets as much as probable before the node energy is exhausted. The residual energy of the node can be calculated after the maximum data packets are transmitted within the ad hoc boundary.

Suppose the transmission power of the each node can accomplished to adjust dynamically, that the nodes can directly communicate with any node to any node with the requirement. The usual routing algorithm implemented in fully connected network instead of direct link use multi path or multi hop connection. The power transmission control combined with multi cost routing algorithm can support optimize the network performance. Distribution of energy consumes uniformly through the network and also enhances the network performance.

The part of network performance intent to calculate the average packet delay, the mean and the variance of the residual energy, the average number of hops on the paths, packet delivery ratio, packet collisions and the network throughput. Adjustable transmission power of nodes and multicost routing algorithm creates exact application routing possible and allows the use of metrics and not possible to considered in singlecost routing.

On the network simulation, a analytically simulation shows the projected multicost routing algorithm decrease the network interference and energy consumption.

Previous work: In general, the routing techniques for ad-hoc networks designed for selecting paths in a network along which to send the data packets in network traffic and variety of performance. Practically all of these routing protocols measured energy efficiency as the decisive objective so as to maximize the entire network lifetime. The routing algorithm designed for to demonstration of small end to end delay between source destination pair, capable of being adopted, effectively usage of the bandwidth, minimum number of data transmissions and also optimize other conditions. All these routing algorithms are single cost in the logic that they consign a scalar cost parameter to every link and compute the path that has minimum cost.

This paper to emphasis on multicost routing, a vector of cost parameters is consigned to each link and the cost vector of a path is well-defined based on the cost vectors of the links that embrace it. (Ephremides, 2002) (Perkins and Bhagwat, 1994) (Perkins and Royer, 1999) presented certain distinguished routing algorithms for wireless ad hoc networks where the metric enhanced are the hop count or end to end delay. (Johnson and Maltz, 1996) used the link quality as the cost metric for routing, (Karagiorgas, et al., 2010) used the ETX as metric which integrates the link loss proportions and the interference between successive links of a network path.

(Rodoplu and Meng, 1999) enormously work has been proposed and developed very first on energy aware routing for wireless ad hoc networks and vital role for develop the energy efficient routing. In (Chang and Tassiulas 2000) and (Chang and Tassiulas, 2004) link costs are calculated based on the energy consumption for unit flow communication and the initial and residual energy at the communicating nodes. In (Gupta and Das, 2002) a cost metric uses the left over battery level and also the number of neighbors of a node. Proposed cost metric used as transmission power control (Karagiorgas et al., 2007) to succeed efficient energy usage. For the calculation of the node transmission power (Banerjee and Misra 2002) two algorithms are suggested. 
In (Chang and Tassiulas, 2000) a distributed algorithm is proposed that increases the node life spans. Further works integrate with power control in the MAC layer (Manohar and Scaglione, 2003). The slow start MAC protocol is suggested in (Shah and Rabaey, 2002), Power control is merged in the MAC layer by with the RTS-CTSDATA-ACK sequence to reach an arrangement on the transmission power. Joint power control (Li, et al., 2007) scheduling and routing algorithms are offered.

Furthermore, a number of devices be present capable of adjusting dynamically their transmission power, such as the Sun SPOT devices from sunspot world. Stated above the routing protocols of cost metrics mentioned for single and scalar metric.

In the face of the possible of multicost routing, the research doings on this field has not been concentrated. The idea of multicost routing was presented in (Papageorgiou et al., 2006) where it was implemented to wire line max-min fair share networks.(Gutierrez, et al., 2000) has investigated multicost routing in wireless ad-hoc networks, In (Karagiorgas et al., 2010) proposes the multicost routing with variable transmission power for enhancing wireless ad hoc network performance.

\section{MATERIALS AND METHODS}

\section{Multicost routing algorithms:}

Multicost routing: In wireless ad hoc networks mobile nodes are linked with dominated path. In the multicost routing algorithm links and paths constructed with cost vector parameters, each links are categorized by cost vector parameters and path cost vector is constructed on cost vectors of links on the network. In addition cost vector is defined for the entire path created on the cost vectors of its necessary links.

The process of path detection progression, the path detected to be non-dominated by another path relating to all cost components are rejected and ignored later. At last, after the optimal path selection from path detection progress the cost vectors of the path assigned with optimization function. The simplified form of multiconstraint problem (Papageorgiou et al., 2006) is multicost problem with no restrictions occur.

The parameters of a link in single cost routing are merged as a single metric in the network, based on this features single cost routing limits with measures that can be used as routing and cannot provide the best of quality of service in different level of the network. Whereas, the cost vector parameters of multicost routing is treated independently until the very end, when an optimization function is applied. Furthermore, in single cost routing the cost parameters maintained as just one path, but in multicost routing maintained a set of no dominated paths for the entire source to destination nodes.

In multicost routing, (Karagiorgas et al., 2010) a vector of cost parameters is consigned to each link and the cost vector of a path is well-defined based on the cost vectors of the links that combined it it by applying, component wise and uses a monotonic associative operator $\odot$ to all the cost vector parameter. Path cost vector is constructed on cost vectors of links on the network are included with the associative operator used for entire cost vector components and by condition applied to them (to maximize or minimize) for choose the optimum path.

In the detail specification, the link cost vector parameter of Link 1 is represent by $\mathrm{V}(\mathrm{P})=\left(\mathrm{V}_{11}, \mathrm{~V}_{21}, \ldots\right.$, $\mathrm{V}_{\mathrm{kl}}$ ) and the cost vector of the path $\mathrm{P}$ in represent by $\mathrm{V}$ $(\mathrm{P})=\left(\mathrm{V}_{1}, \mathrm{~V}_{2}, \ldots, \mathrm{V}_{\mathrm{k}}\right)$ contains of links $1=1,2 \ldots 1$. The optimization function $\mathrm{f}(\mathrm{V})$ has to be minimized so as to choose the optimum path. The path cost vector $\mathrm{V}(\mathrm{P})=$ $\left(\mathrm{V}_{1}, \mathrm{~V}_{2}, \ldots, \mathrm{V}_{\mathrm{k}}\right)$ contains of links $1=1,2 \ldots 1$ is derived from link cost vector $\mathrm{V}(\mathrm{P})=\left(\mathrm{V}_{11}, \mathrm{~V}_{21}, \ldots, \mathrm{V}_{\mathrm{kl}}\right)$ that comprise it along with representing of component-wise a monotonic associative operator $\odot$ to all the cost vector parameter Eq. 1:

$\mathrm{V}_{\mathrm{m}}=\odot_{\mathrm{l}=1}^{\mathrm{L}} \mathrm{V}_{\mathrm{ml}}$

The dissimilar cost vector components might have different monotonic associative operator The path cost vector of determined from link cost vector that comprise it along with representing of component-wise a monotonic associative operator $\odot$ to all the cost vector parameter and by condition applied to them (to maximize or minimize) for choose the optimal path indicated in Eq. 1. Below indicated equations Eq. 2-4 are $\mathrm{m}^{\text {tn }}$ parameter of the cost vector type namely Additive, Restrictive and Maximum representative.

- Additive, where

$\mathrm{V}_{\mathrm{m}}=\sum_{\mathrm{l}=\mathrm{l}}^{\mathrm{L}} \mathrm{V}_{\mathrm{ml}}, \mathrm{V}_{\mathrm{ml}} \geq 0$

Proposed objective is to decrease the $V_{m}$ and $f$ is monotonically growing in $\mathrm{V}_{\mathrm{m}}$,

- Restrictive, where

$\mathrm{V}_{\mathrm{m}}=\min _{\mathrm{l}=1, \ldots, \mathrm{L}}\left\{\mathrm{V}_{\mathrm{ml}}\right\}$ 
Proposed objective is to increase the $\mathrm{V}_{\mathrm{m}}$ and $\mathrm{f}$ is monotonically reducing in $\mathrm{V}_{\mathrm{m}}$ then

-Maximum representative, where

$$
\mathrm{V}_{\mathrm{m}}=\max _{1=1, \ldots, \mathrm{L}}\left\{\mathrm{V}_{\mathrm{ml}}\right\}
$$

Projected objective is to decrease $\mathrm{V}_{\mathrm{m}}$ and $f$ is monotonically reducing in $\mathrm{V}_{\mathrm{m}}$

A multicost routing algorithm contains of two segments. In the first segment, execute the Dijkstra's algorithm in certain time period and acquired a set of non-dominated path from source to destination nodes of the ad hoc networks. Non-dominated path, using some cost standards, not possible to find other better path and using some other cost standards presence worse to find better path. A complete explanation of the algorithm is described in (Gutierrez et al., 2000) and this segment decreases expressively the algorithm's total calculation attempt.

In the second segment, the source node desires to route a packet to a used destination node a scalar cost function $\mathrm{f}$ is assigned to the cost vectors of the nondominated paths directing to that destination node and the path that the minimum cost is selected done by multicost routing algorithm. The optimization function freely on the Quality of Service requirements of the session and it will vary for dissimilar session.

Cost parameters for ad hoc networks: In the projected multicost routing algorithm for wireless ad hoc network consists of the following five parameters. The number of hops $\mathrm{h}$ in a path (Eq. 5), the total interference $I_{1}$ (Eq. 7), the node link delay $d_{1}$ of a path (Eq. 8), the minimum residual energy $\mathrm{R}$ of a path (Eq. 9) and the transmission powers T (Eq. 10), used by the nodes on a path.

The number of hops $h$ in a path:

$\mathrm{h}=\sum_{\mathrm{l}=1}^{\mathrm{j}} \mathrm{h}_{1}$

where, $h_{l}=1$ for all links 1 , the associative operator $\odot$ used here for addition.

The total interference $I_{1}$, or the maximum interference $\mathrm{I}_{\infty}$ produced by using a path: The number of nodes inside the communication range of the source and destination nodes of link 1 are cause of total interference. Where $a$ and $b$ is transmitter and receiver of link $1=(\mathrm{a}, \mathrm{b})$ as $|\mathrm{a}, \mathrm{b}|$, then it becomes Eq. 6 :

$$
\mathrm{I}_{1}=\mathrm{I}_{(\mathrm{a}, \mathrm{b})}=|\{\mathrm{c} \in \mathrm{V},|\mathrm{b}, \mathrm{c}| \leq|\mathrm{a}, \mathrm{b}|\} \mathrm{U}\{\mathrm{c} \in \mathrm{V},|\mathrm{a}, \mathrm{c}| \leq|\mathrm{a}, \mathrm{b}|\}|-2
$$

The total interference $I_{1}$ and $I_{\infty}$ both are usually desirable. Total interference $\mathrm{I}_{1}$, measured from combined in the link metrics by applying minimization additive operator and $\mathrm{I}_{\infty}$ is measured from combined in the link metrics by applying maximization additive operator:

$l_{1}=\sum_{1=1}^{j} I_{1}$ (or) $I_{\infty}=T_{\infty}=\max _{1=1, \ldots, j} I_{1}$

The node link delays $d_{l}$ or equivalently, its length $d_{t}$ (Scalar) where $d_{l}=1$ for all links 1 :

$\mathrm{d}=\sum_{\mathrm{l}=1}^{\mathrm{j}} \mathrm{d}_{1}$

Transmitting for peer to peer nodes constructed on link delay, for easiness to take on the link delay limit $d_{1}$ is equal to $2^{\mathrm{k}} * \mathrm{~d}$, where $\mathrm{d}$ is delay base and $\mathrm{k}$ is delay exponent of a node. The total delays of link $d_{1}$ or the maximum link delay $\mathrm{d}_{\infty}$ is caused by using a path.

The minimum residual energy $\mathrm{R}$ of a path:

$\mathrm{R}=\min \mathrm{R}_{1}$

The transmitting nodes residual energy of link $R_{1}$ is one of the link cost metric. Using minimization operator to the link acquired minimum residual energy $\mathrm{R}$ is normally desirable. It denotes degree of energy critical path.

The minimum $T_{1}$ or the maximum $T_{\infty}$ of the transmission powers acquired from the nodes on a path:

$$
\mathrm{T}_{1}=\sum_{\mathrm{i}=1}^{\mathrm{j}} \mathrm{T}_{1}
$$

or

$\mathrm{T}_{\infty}=\max _{\mathrm{l}=1, \ldots, \mathrm{j}} \mathrm{T}_{1}$

The parameters only captures the current energy state of the network and as a result also used the transmission power $T_{1}$ is mandatory for exact reception in the link 1. $\mathrm{T}_{1}$ is measured from conjoining the link metrics by applying minimization additive operator whereas $\mathrm{T}_{\infty}$ is measured from conjoining the link metrics by applying maximization additive operator. $T_{1}$, $\mathrm{T}_{\infty}$ both are desirable when $\mathrm{T}_{1}$ consumes less amount of energy from overall energy and path with small amount of $\mathrm{T}_{\infty}$ avoid energy critical nodes.

The number of hops $h$ in $a$ path(minimum/maximum), The total interference 1 of a 
path (minimum/maximum), The node link delays $\mathrm{d}$ (maximum/minimum), The transmitting nodes residual energy of link $\mathrm{R}$ (minimum/maximum) and Transmission power $\mathrm{T}$ (maximum/minimum) are the five cost parameters to make clear the view of control in the dominate of path.

From which the path $\mathrm{p} 1$ along with the cost vectors $\mathrm{V} 1=\{\mathrm{h} 1, \mathrm{I} 1, \mathrm{~d} 1, \mathrm{R} 1, \mathrm{~T} 1\}$ and $\mathrm{p} 2$ along with the cost vectors $V 2=\{h 2, I 2, d 2, R 2, T 2\}$ which is to dominate the path $\mathrm{p} 1$, when $\mathrm{h} 1<\mathrm{h} 2, \mathrm{I} 1<\mathrm{I} 2, \mathrm{~d} 1<\mathrm{d} 2, \mathrm{R} 1>\mathrm{R} 2$ and $\mathrm{T} 1<\mathrm{T} 2$.

The path p1 may dominate when $\mathrm{h} 1<\mathrm{h} 2, \mathrm{I} 1>\mathrm{I} 2$, $\mathrm{d} 1>\mathrm{d} 2, \mathrm{R} 1<\mathrm{R} 2$ and $\mathrm{T} 1<\mathrm{T} 2$ are showed less interference due to spent little amount of transmission power with same number of hops and reverse of residual and link delay. The path $\mathrm{p} 1$ will be a non-dominated path when it is not dominated by some other path.

Meanwhile energy of a network and less interference of a path is significant role in the wireless ad hoc networks. Thus, the transmission power and interference cost metrics are included in the proposed cost parameters as well as in optimization function. Similarly link delay may increase due to interference and residual energy indicates consume of overall energy in used path.

Optimization functions: Proposed multicost routing algorithms: Based on algorithm the optimization results provide the different significance on the various parameters (dependent). The top choice of the optimization function gets from performance evaluations. The optimization function would not perform better though the parameters were independent. MAX/MIN Energy-Half-Interference- Half -Hop algorithm is the best one got from performance comparison of all relevant algorithm.

The proposed cost metrics which are already mentioned are combined in dissimilar method to create a number of multicost routing algorithms.

The Table 1 covers the optimization functions inspected, all corresponding to a various multi cost routing algorithm for picking the paths. Even if the parameters were independent, this would not help us know a priori which optimization function would perform better.

Adjustable transmission power: The capability of mobile transmission nodes to adjust transmission power (Agarwal et al., 2001) themselves is most essential. This capability is not present in the fixed transmission and therefore even if enlarge the nodes with enormous amount of energy and not to lead unnecessary interference to other nodes. Whereas in mobile nodes transmission if enlarge the nodes with enormous amount of energy and it leads to unnecessary interference to other nodes.
Table 1: The multicost routing algorithms

\begin{tabular}{ll}
\hline Name & Optimization function \\
\hline Minimum Interference & $\mathrm{I}_{1}(\mathrm{P})$ \\
MAX Interference & $1_{\propto}(\mathrm{P})$ \\
Minimum Transmission & $\mathrm{T}_{1}(\mathrm{P})$ \\
Power & \\
SUM/MIN Energy- & $\frac{\mathrm{T}_{1}(\mathrm{P}) \cdot \mathrm{I}_{1}(\mathrm{P})}{\mathrm{R}(\mathrm{P}) \cdot \mathrm{d}(\mathrm{P})}$
\end{tabular}

Interference

SUM/MIN Energy-

$\frac{\sqrt{h(P)} \cdot T_{1}(P) \cdot I_{1}(P)}{R(P) \cdot d(P)}$

Interference-Half Hop

MAX/MIN Energy -

$\frac{T_{\infty}(P) \cdot I_{\infty}(P)}{R(P) \cdot d(P)}$

Half- Interference

MAX/MIN Energy-Half-

$$
\frac{\sqrt{h(P)} \cdot T_{\infty}(P) \cdot \sqrt{I_{\infty}(P)}}{R(P) \cdot d(P)}
$$

Interference -Half Hop

The capability of mobile transmission nodes to adjust transmission power themselves which outcomes shows quandaries and compromises. The proposed multicost algorithm mainly tends to solve the capability of mobile transmission nodes to adjust transmission power. For example, , in Fig. 2 node 'a' may communicate directly to node 'f', or communicate through b, c, d and e nodes as intermediate relays. From the first transmission total number a hop is sum of one, but consume huge amount of transmission power. Whereas in the second transmission total hop count is four even with less transmission power consumed than first transmission.

In another example showing benefits gained from the flexibility supporting by the adjustable transmission power in the Fig. 3. In Fig. 3b inability to communicate with nodes $\mathrm{C}$ and $\mathrm{D}$ due to the node $\mathrm{C}$ presented in reserved area of A and B (802.11 MAC protocol). And in Fig. 3a nodes $\mathrm{C}$ and $\mathrm{D}$ can communicate with each other also adjusts their transmission power to the minimum required.

The network topology which is not fixed and it is based on the transmission powers chosen because of mobility nodes have capability adjust their transmission power. During the execution of the algorithm and the candidate paths construction stage, all the different combinations of nodes transmission power level also lead to network topologies are valued. Caused by the domination relations applied some of these combinations are rejected from additional consideration, decreasing this way the solution space.

Perhaps, in Fig. 2 multicost algorithm, for the duration of the path phase, found two paths linking nodes ' $a$ ' to ' $f$ ' are (1) (a, f) and (2) (a, b, c, d, e, f). The node ' $a$ ' has $\mathrm{T}_{\mathrm{a}}^{\mathrm{large}}$ and $\mathrm{T}_{\mathrm{a}}^{\text {small }}$ of two various transmission power levels maintained. 


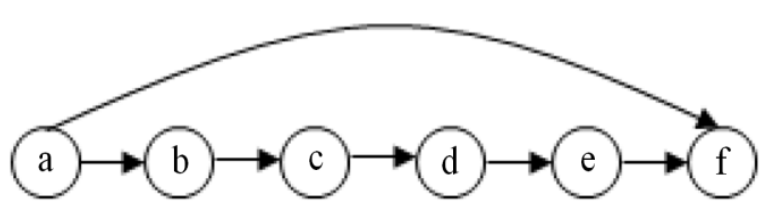

Fig. 2: First example of adjustable transmission power

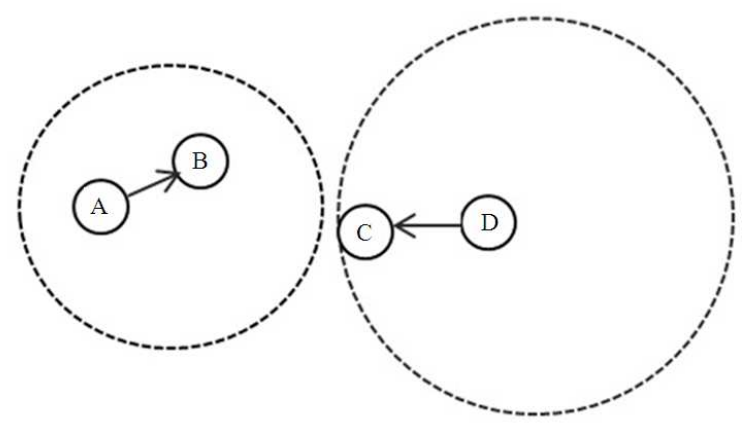

(a)

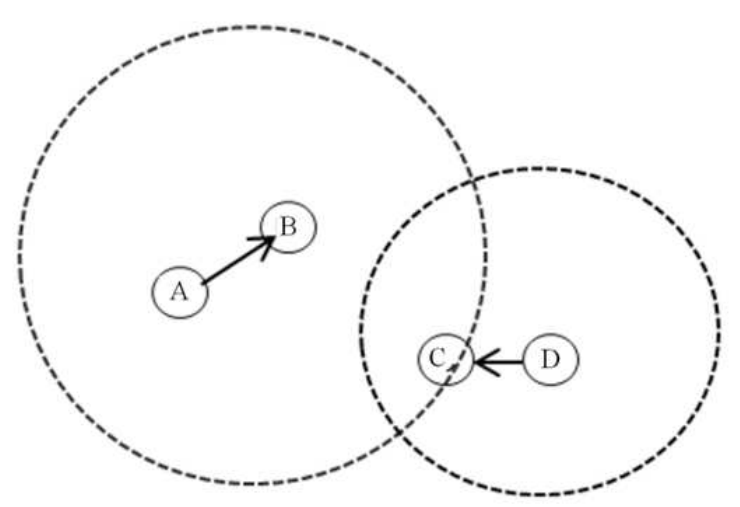

(b)

Fig. 3: Second example of adjustable transmission power

The same way used three cost metrics for path (1) and (2): $\left(T_{a}^{\text {large }} R_{a}, I_{a}\right)$ and $\left(T_{a}^{\text {small }} R_{a}, I_{a}+T_{b}+T_{c}+T_{d}+, \min \left\{R_{a}\right.\right.$, $\left.\mathrm{R}_{\mathrm{b}}, \mathrm{R}_{\mathrm{c}}, \mathrm{R}_{\mathrm{d}}, \mathrm{R}_{\mathrm{e}}\right\}, \min \left\{\mathrm{d}_{\mathrm{a}}, \mathrm{d}_{\mathrm{b}}, \mathrm{d}_{\mathrm{c}}, \mathrm{d}_{\mathrm{d}}, \mathrm{d}_{\mathrm{e}}\right\}, \max \left\{\mathrm{I}_{\mathrm{a}}, \mathrm{I}_{\mathrm{b}}, \mathrm{I}_{\mathrm{c}}, \mathrm{I}_{\mathrm{d}}\right.$, $\left.I_{e}\right\}$ ), This way there are three cost vectors for paths (1) and (2): $\quad\left(\mathrm{T}_{\mathrm{a}}^{\text {large }} \mathrm{R}_{\mathrm{a}}, \mathrm{I}_{\mathrm{a}}\right) \quad$ and $\left(T_{a}^{\text {small }} R_{a}, I_{a}+T_{b}+T_{c}+T_{d}+, \min \left\{R_{a}, R_{b}, R_{c}, R_{d}, R_{e}\right\}\right.$, $\left.\min \left\{d_{a}, d_{b}, d_{c}, d_{d}, d_{e}\right\}, \max \left\{I_{a}, I_{b}, I_{c}, I_{d}, I_{e}\right\}\right)$, assuming additive, minimization and maximization operators for the transmission power $\mathrm{T}$, the residual energy $\mathrm{R}$, node delay of the link $\mathrm{d}$ and the interference I parameters, respectively.

Then to check these path dominance relations. If, there is no path domination to continue with applying an optimization function with respect to select from one of them available also select the equivalent transmission power of node ' $a$ '. Else, in case of topology in Fig. 2 proceed to extending these two subpaths consistent with the multicost approach and considering node ' $a$ ' small and large transmission power levels.

Simulation model: The projected multicost routing algorithm calculated through network evacuation pattern. The network communication begins with some amount of energy to carry some packets in the candidate path and deliver as much packets as probable before the energy at the nodes is exhausted. Executed this projected multicost algorithm using NS2 network simulator.

Basically routing process consist two stages, the routing information exchange and the routing algorithm. Routing information exchange protocols deal with gathering and broadcasting network stage information and routing algorithms calculate the best optimal path based on first stage information. This paper concentration on the stage of routing algorithm and hence assumes that all the nodes have global knowledge of the network topology and all other information it wants to take routing decisions.

Certain choosing of the optimization function the multicost routing difficult to distributed implementation if source routing is used. When a data packet is produced at a node and the node applies the optimization function for combined the cost metrics with respect to the non-dominated path to choose the suitable path and the packet is sent on that optimal path. If the node could not be found the destination route then the data packet to be disposed. The model ad hoc network simulated comprises with 50 nodes static unsystematically located in the $400 \times 400 \mathrm{~m}^{2}$ area.

The threshold of the received signal's power necessary for accurate reception is the similar for all nodes. In the tryout of simulation the capability of mobile transmission nodes to energetically adjusting transmission power themselves. And take on that the network is completely connected with each other and communicate directly. But, activation of routing algorithm a source node may choose multipath instead of direct link.

The nodes may recognize the network topology and found physical distance with other nodes. Thus the nodes can alter their transmission power. So that they can adjust their transmission power to the minimum value required for coherent reception at the reception end, so as to consume only the minimum required energy and may form less interference.

Actually, most of the presently available devices afford a limited set of possible power levels of transmission power control. Assume that the transmission power can take sequence values are mainly made for the simulations and is not essential by the algorithms themselves. To calculate the minimum 
transmission power for communicate among two nodes with some distance $\mathrm{d}$ can use the following equation mentioned below in Eq. 11 .

$$
P_{r}(D)=\frac{P_{t} \cdot G_{t} \cdot G_{r} \cdot \lambda^{2}}{(4 \pi)^{2} \cdot D^{a} \cdot L}
$$

where, $P_{r}$ is the received power signal, $P_{t}$ is the transmitted power signal $G_{t}$ is the gain the senders antenna, $G_{r}$ is the gains of receivers antenna, $L \geq 1$ the system loss and $\lambda$ the wavelength used. In the calculations, assume $L=1$ and $G_{t}=1, G_{r}=1$.The parameter ' $a$ ' is the path loss constant and is usually in between 2 and 4 subject to the wireless channel as well as assume $\mathrm{a}=2$, corresponding to the Free Space transmission model.

This is because of the interference restricted environment, where effective reception is based on the signal to interference and noise ratio and the fact that the line of sight transmission $(\mathrm{a}=2)$ between any couple of nodes in any network is commonly rare.

\section{RESULTS}

Performance results: The performance of the projected SUM/MIN Energy-Interference multicost routing algorithm assessed in the simulation experiments. The following performances metrics are measured.

- At the end of every simulation test the average residual energy $\mathrm{E}$ remaining at the nodes to be exact, all the data packets was evacuated from the network.

- The difference $\sigma_{E}^{2}$ of the node residual energies.

- The packets are transmitted in the path on hops and average number of hops is $h$.

- The packet delivery ratio (Receive/Sent), represented by RS. It is calculated by dividing the number of data packets received by the destination through the number of data packets originated and supposed to transmit all the data packets before a node lost the energy.

- The number of data packet collisions $\mathrm{C}$ because of the MAC protocol and the hidden terminal problem.

- The average packet delay $\mathrm{D}$, is the average time it takes a data packet to reach the destination.

- The network throughput $\mathrm{T}$ it is measured in bits per second (bit/s or bps) is well-defined as the total amount of data receives from the source divided by the time it takes for the receiver to receive the last packet.
The first two performances calculate the energy concerns, rest other five performances exactly related to network performance.

Performance of MAX/MIN algorithms: The performance of the MAX/MIN algorithms with same cost parameters and network as mentioned earlier. The optimization results shows in the figures regarding with variance of residual energy $\sigma_{E}^{2}$ (Fig. 4) and the packet delivery (Received/Sent ) ratio (Fig. 5) with early finite energy, which is the most accurate and substantial case.

The polynomial MAX/MIN algorithms and exponential SUM/MIN algorithms are examined and show the result. Though, in fact, established that the running times of all the algorithms were conventional, at best for the network sizes used in the simulations. And compared both the MAX/MIN Energy- HalfInterference and the MAX/MIN Energy- HalfInterference- Half Hop algorithms with relevant to SUM/MIN algorithms.

SUM/MIN Energy-Half-Interference-Half Hop algorithm is similar optimization function with MAX/MIN Energy-Half-Interference-Half Hop algorithm excluding that the transmission power (maximum) and the interference (maximum) in place of additive cost metrics.

The overall performance of the SUM/MIN algorithms and relevant with the MAX/MIN algorithms, The SUM/MIN algorithms are shows improved performance over the MAX/MIN algorithms.

By the way of explanation, $\mathrm{T}_{1}$ and $\mathrm{L}_{1}$ metrics are more suitable than $T_{\propto}$ and $I_{\propto}$ metrics correspondingly in creating routing decisions and for the adding up of the values of the transmission path the $T_{1}$ and $L_{1}$ metrics are the best representative metric over $\mathrm{T}_{\propto}$ and $\mathrm{I}_{\propto}$ metrics. Note that if $\mathrm{T}_{1}$ and $\mathrm{L}_{1}$ metrics are applied the SUM/MIN algorithm gets exponential complexity whereas the $\mathrm{T}_{\propto}$ and $\mathrm{I}_{\propto}$ metrics are applied it becomes polynomial.

Comparison of SUM/MIN Energy-Interference with a singlecost algorithm: The simulation tests are conducted number of times and performances compared multicost SUM/MIN Energy-Interference algorithm with single cost algorithm. The cost of the each link in single cost algorithm is $\frac{T . I}{R}$, where $T$ is the link of transmission power of source node, I is the link interference metrics and $\mathrm{R}$ is the residual energy of the link of destination node. In figure 5 shows the packet delivery (Received/Sent) ratio at the end of an evacuation problem in finite initial energy. 

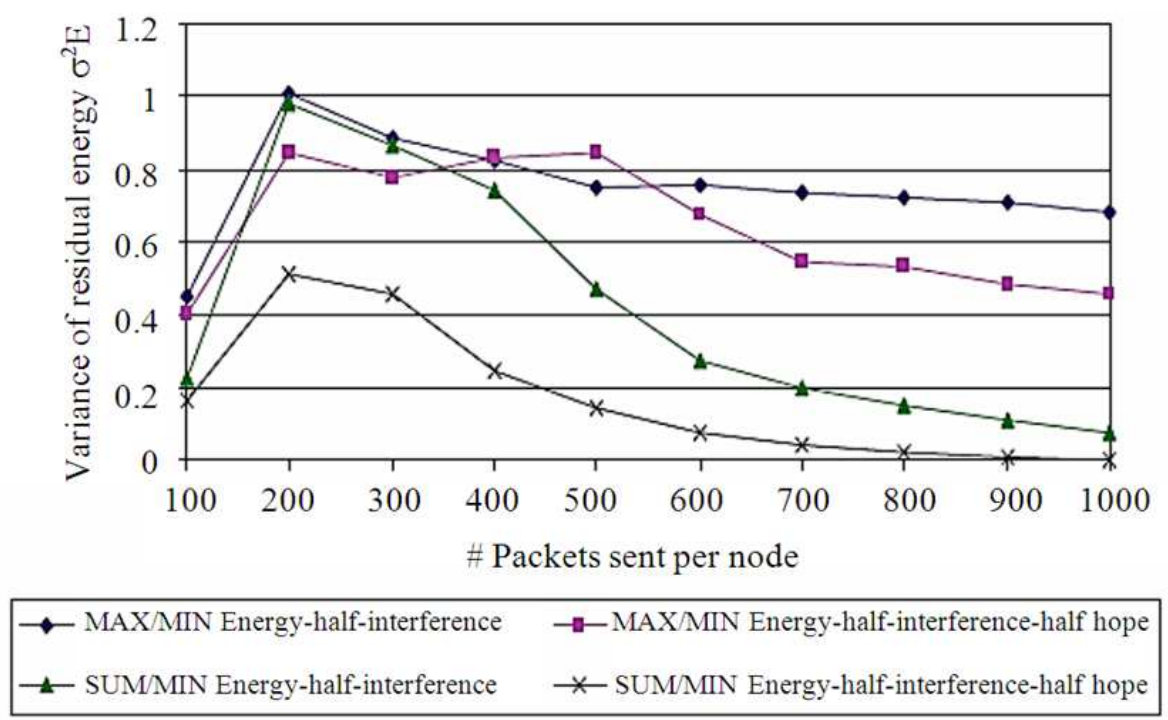

Fig. 4: Illustrate the variance of the residual energy at the end of an evacuation problem,different choices of the routing algorithms

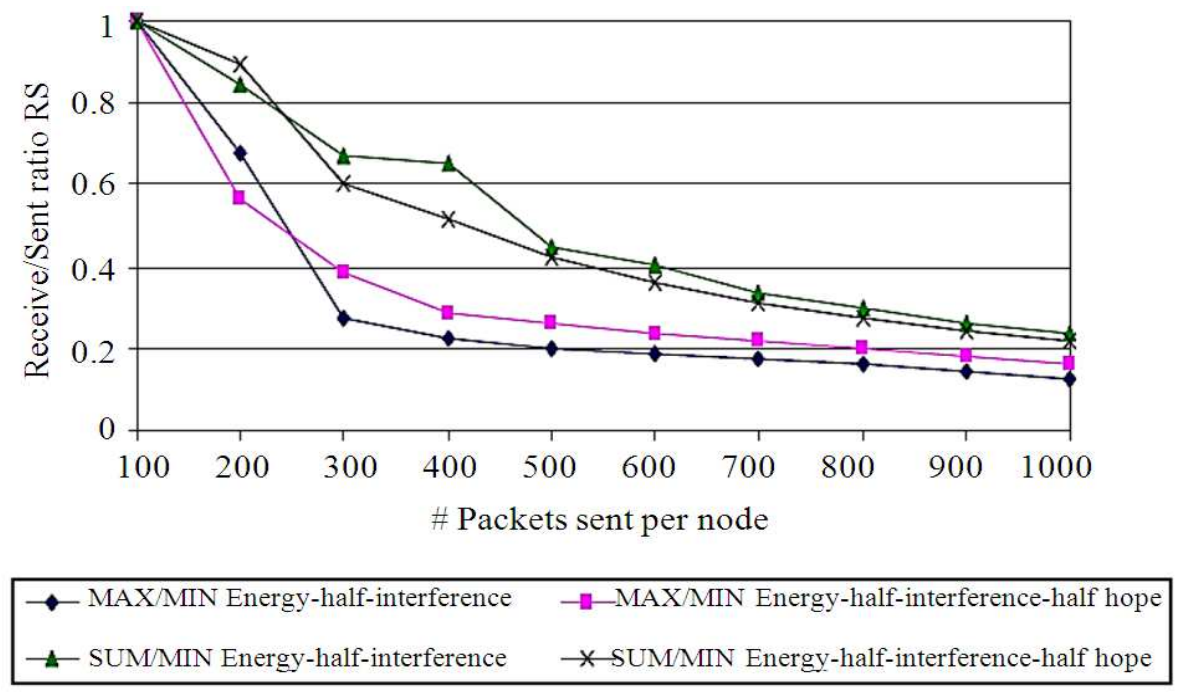

Fig. 5: Illustrates the Packet delivery (Received / send) ratio at the end of an evacuation problem, as a function of the number of packets evacuated per node, for the case of finite initial energy

Observed from the graph Fig. 6 the multicost SUM/MIN Energy-Interference algorithm is dynamically and effectively delivered the packets to the destination node. Since the multicost SUM/MIN Energy-Interference algorithm well usage of the nodes energy reserves and efficiently delivered the packets. The multicost SUM/MIN Energy-Interference algorithm maintaining longer time energy than singlecost algorithm. And also multicost algorithm spends to distribute over a region the energy consumption consistently in the network. Obviously, if a node is working in out of energy all other the equal energy and in crucial state.

Also noted that both the multicost and singlecost algorithm after some extended time instances few nodes had a zero energy and the network considered as disconnected. If nodes trying to transmit new packets during this zero energy, fail to communicate and occurred packet dropped. The multicost SUM/MIN Energy- Interference algorithm performs well is that it better encapsulates the significance of all the cost parameters in the entire path. 


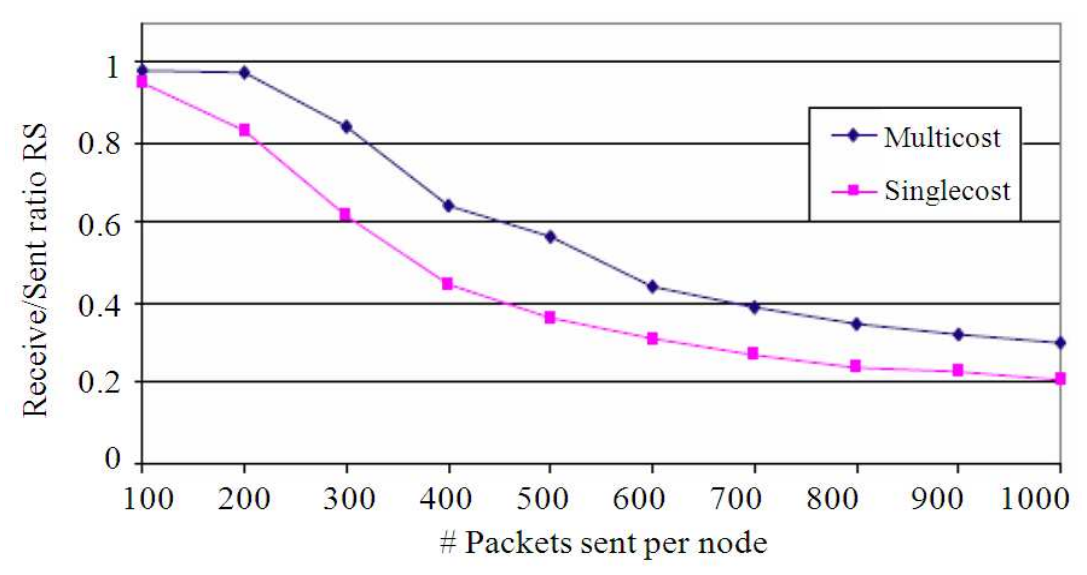

Fig. 6: Illustrate a received to send ratio at the end of an evacuation problem as a function of the number of packets evacuated per node in the multicost SUM/MIN Energy-Interference and corresponding with singlecost algorithm

Still, analyzed a lot of advantages are there using the multicost methodology and it can be measured quantitatively. One of the most standard of these advantages are likely to run the algorithm and then choose path using several optimization function simultaneously depends on need of quality service. Such as one of the examples is a node can route to transmit the new packets in a path using the multicost SUM/MIN Energy-Interference algorithm at the similar time involved in SUM/MIN Energy-Half-Interference optimization functions. Whereas in the single cost routing to obtain the above result has to re execute number of times using various link cost parameters in every time.

Similarly, in single cost approach there is not possible to hop count in a particular path. But in the multicost approach straightly accounted to adding hop count parameter in link cost vector and it is implemented in SUM/MIN Energy-Interference-Hop algorithm.

\section{DISCUSSION}

Since the singlecost routing is scalar cost parameters were consigned to all the link and path that has single cost measure and minimum cost. It is used to find the optimal path and which can be used for routing and cannot provide the different QoS levels of ad hoc network.

Whereas in multicost routing a vector of cost parameters is consigned to each link and the cost vector of a path is well-defined based on the cost vectors of the links that combined it by applying, component wise and uses a monotonic associative operator to all the cost vector parameters and adjustable transmission power of the mobile nodes which leads to obtain the dynamic energy and improves the QoS of wireless ad hoc network.

\section{CONCLUSION}

This proposed multicost routing approach a vector of cost parameters is consigned to each link and the cost vector of a path is defined based on the cost vectors of the links that embrace it with the adjustable transmission power and it leads to ad hoc networks performance. The projected multicost algorithm is entirely different form single cost routing. Multicost routing supports inspect and implement in various algorithms and all optimizing a dissimilar cost function and evaluated the power adjustment ability of the nodes. All the cost parameters like hop count, total interference, node link delay, residual energy of a node and the node transmission power were measured and their effect was calculated.

Performance evaluated the proposed multicost SUM/MIN Energy-Interference routing algorithms conducted a number of energy and interference experiments provides the most balanced results related to the network performance like reduced interference and improve packet delivery (Receive/Sent) ratio. At last, the function optimized that the multicost SUM/MIN Energy-Interference algorithm with the adjustable transmission power results showed better than the singlecost algorithm in wireless ad hoc networks.

\section{REFERENCES}

Agarwal, M., S. Rajani and A. Mishra, 2001. Study of flocculation efficiency of okra gum in sewage wastewater. Macromol. Mater. Eng., 286:560-563.

Banerjee, S. and A. Misra, 2002. Minimum energy paths for reliable communication in multi-hop wireless networks. Proceedings of the 3rd ACM International Symposium on Mobile ad Hoc Networking and Computing, Jun. 9-11, ACM Press, USA, pp: 146-156. DOI: $10.1145 / 513800.513818$ 
Chang, J.H. and L. Tassiulas, 2000. Energy conserving routing in wireless ad-hoc networks. Proceedings of the IEEE 19th Annual Joint Conference of the IEEE Computer and Communications Societies, Mar. 26-30, IEEE Xplore Press, Tel Aviv, pp: 2231. DOI: $10.1109 /$ INFCOM.2000.832170

Chang, J.H. and L. Tassiulas, 2004. Maximum lifetime routing in wireless sensor networks. IEEE/ACM Trans. Netw., 12: 609-619. DOI: 10.1109/TNET.2004.833122

Ephremides, A., 2002. Energy concerns in wireless networks. IEEE Wireless Commun., 9: 48-59. DOI: 10.1109/MWC.2002.1028877

Gupta, N. and S.R. Das, 2002. Energy-aware ondemand routing for mobile ad hoc networks. Distributed Comput., 2571: 164-173. DOI: 10.1007/3-540-36385-8_17

Gutierrez, F.J., E. Varvarigos and S. Vassiliadis, 2000. Multicost routing in max-min fair share networks. Department of Electrical Engineering.

Johnson, D.B. and D.A. Maltz, 1996. Dynamic source routing in ad hoc wireless networks. Mobile Comput., 353: 153-181. DOI: 10.1007/978-0-58529603-6_5

Karagiorgas, N.M, P.C. Kokkinos, C.A. Papageorgiou and E.A. Varvarigos, 2007. Multicost routing in wireless AD-HOC networks with variable transmission power. Proceedings of the IEEE 18th International Symposium on Personal, Indoor and Mobile Radio Communications, Sept. 3-7, IEEE Xplore Press, Athens, pp: 1-5. DOI: 10.1109/PIMRC.2007.4394717

Karagiorgas, N., P. Kokkinos, C. Papageorgiou and E. Varvarigos, 2010. Joint multi-cost routing and power control in wireless ad hoc networks. Wireless Networks, 16: 2263-2279. DOI: 10.1007/s11276-010-0257-z

Li, Y. and A. Ephremides, 2007. A joint scheduling, power control and routing algorithm for ad hoc wireless networks. Ad Hoc Networks, 5: 959-973. DOI: 10.1016/j.adhoc.2006.04.005
Li, Z., J. Li, W.P. Menzel, T.J. Schmit and S.A. Ackerman, 2007. Comparison between current and future environmental satellite imagers on cloud classification using MODIS. Remote Sensing Environ., 108: 311-326. DOI: 10.1016/j.rse.2006.11.023

Manohar, R. and A. Scaglione, 2003. Power optimal routing in wireless networks. Proceedings of the IEEE International Conference on Communications, May 11-15, IEEE Xplore Press, pp: 2979 -2984 . DOI: 10.1109/ICC.2003.1204613

Papageorgiou, C.A., P.C. Kokkinos and E.A. Varvarigos, 2006. Multicost routing over an infinite time horizon in energy and capacity constrained wireless ad-hoc networks. Euro-Par., 4128: 931-940. DOI: 10.1007/11823285_98

Perkins, C.E. and E.M. Royer, 1999. Ad-hoc ondemand distance vector routing. Proceedings of the 2nd IEEE Workshop on Mobile Computing Systems and Applications, Feb. 25-26, IEEE Xplore Press, New Orleans, LA, pp: 90-100. DOI: 10.1109/MCSA.1999.749281

Perkins, C.E. and P. Bhagwat, 1994. highly dynamic destination-sequenced distance-vector routing (DSDV) for mobile computers. Proceedings of the Conference on Communications Architectures, Protocols and Applications, (CAPA' 94), ACM Press, USA, pp: 234-244. DOI: 10.1145/190809.190336

Rodoplu, V. and T.H. Meng, 1999. Minimum energy mobile wireless networks. IEEE J. Selected Areas Commun., 17: 1333-1344. DOI: 10.1109/49.779917

Shah, R.C. and J.M. Rabaey, 2002. Energy aware routing for low energy ad hoc sensor networks. Proceedings of the IEEE Wireless Communications and Networking Conference, Mar. 17-21, IEEE Xplore Press, pp: 350-355. DOI: 10.1109/WCNC.2002.993520 